\title{
Autonomous Uncertainty Quantification for Discontinuous Models Using Multivariate Pad Approximations
}

\author{
Richard Ahlfeld* \\ Uncertainty Quantification Lab \\ Department of Aeronautics \\ Imperial College London \\ UK
}

\author{
Mauro Carnevale \\ Osney Thermo-Fluids Laboratory \\ Department of Engineering Science \\ University of Oxford \\ Oxford OX2 0ES, UK
}

\author{
Simone Salvadori \\ Department of Industrial Engineering \\ University of Florence \\ Florence \\ Italy \\ Francesco Montomoli \\ Head of Uncertainty Quantification Lab \\ Department of Aeronautics \\ Imperial College London \\ UK
}

\begin{abstract}
Problems in turbomachinery Computational Fluid Dynamics (CFD) are often characterised by non-linear and discontinuous responses. Ensuring the reliability of Uncertainty Quantification (UQ) codes in such conditions, in an autonomous way, is a challenging problem. In this work, we suggest a new approach that combines three state-ofthe-art methods: multivariate Padé approximations, Optimal Quadrature Subsampling and Statistical Learning. Its main component is the generalised least squares multivariate Pad -Legendre (PL) approximation. PL approximations are globally fitted rational functions that can accurately describe discontinuous non-linear behaviour. They need fewer model evaluations than local or adaptive methods and do not cause the Gibbs phenomenon like continuous Polynomial Chaos methods. A series of modifications of the Pad algorithm allow us to apply it to arbitrary input points instead of optimal quadrature locations. This property is particularly useful for industrial applications, where a database of CFD runs is already available, but not in optimal parameter locations. One drawback of the PL approximation is that it is non-trivial to ensure reliability. To improve stability we, therefore, suggest to couple it with Optimal Quadrature Subsampling. Our reasoning is that least squares errors, caused by an ill-conditioned design
\end{abstract}

*Address all correspondence to this author, email: r.ahlfeld14@imperial.ac.uk 
matrix, are the main source of error. Finally, we use statistical learning methods to check smoothness and convergence. The resulting method is shown to efficiently and correctly fit thousands of partly discontinuous response surfaces for an industrial film cooling and shock interaction problem using only 9 CFD simulations.

\author{
Nomenclature \\ UQ Uncertainty Quantification \\ PDF Probability Density Function \\ TET Turbine Entry Temperature \\ $M_{B} \quad$ Blowing ratio \\ $M a \quad$ Mach number \\ $\operatorname{Pr}$ Prandtl number \\ $D$ Diameter of the film cooling holes \\ $T$ Temperature \\ $X$ Stream-wise coordinate \\ $Y \quad$ Pitch-wise coordinate \\ $Z$ Span-wise coordinate \\ $\eta_{a w} \quad$ Adiabatic effectiveness \\ $T_{a, \text { rec }}$ Recovery temperature \\ $T_{a, w} \quad$ Adiabatic wall temperature \\ $T_{0, m} \quad$ Total temperature \\ $d$ Number of input random parameters \\ $c(M)$ Number of Polynomial Chaos basis functions \\ $M$ Order of Padé numerator polynomial \\ $L$ Order of Padé denominator polynomial \\ $N$ Number of tensor grid quadrature points \\ $k$ Variable used in Padé approximation \\ $\xi \quad$ Input random variables \\ $\Gamma$ Tensor product array of univariate Gaussian collocation points \\ $w$ Gaussian quadrature weights \\ $W \quad$ Gaussian quadrature weights diagonal matrix \\ $u(\xi) \quad$ Physical model function \\ $P(\xi)$ Enumerator Polynomial Chaos expansion \\ $Q(\xi) \quad$ Denominator Polynomial Chaos expansion \\ $\psi_{j}, \phi_{j} \quad$ Univariate orthogonal Legendre polynomial of order $\mathrm{j}$ \\ $P_{j}, Q_{j} \quad$ Multivariate linear combination of univariate optimal orthogonal polynomials \\ $\hat{p}_{j}, \hat{q}_{j}$ Polynomial Chaos Fourier coefficients \\ $I_{i, j} \quad$ Polynomial multi-index matrix \\ $\rho(\xi)$ Probability Density Function (uniform distribution) \\ $\omega$ Optimal Gaussian integration weights \\ $B, C, D \quad$ System matrices \\ $A \quad$ Design matrix
}

\title{
1 INTRODUCTION
}

Virtual design through modelling and simulation is extremely attractive for the turbomachinery industry, because it reduces the number of required real-life tests. In the era of virtual design (and "digital twins") there is a great need for systematic and automatic ways to quantify design uncertainties. For transonic flow simulations, however, this is still a challenging problem due to strong non-linearity and discontinuous design space bifurcations created by shocks. One of the biggest issues, in the authors' opinion, is bridging the industrial reality with theoretical advancements made in recent years. While the mathematical theory has been improved greatly, many methodologies are hard to apply to actual test cases. The most often occurring problem is that often only a very low number of CFD model runs are available, - far below any mathematically reasonable convergence requirement. Another frequent problem is that the model has not been evaluated in optimal collocation points. In the mathematical theory, optimal evaluation points are routinely used. In reality, however, there are problems - either due to convergence issues with the CFD solver - or simply because certain configurations were already performed by the CFD engineer. In general, already generated data should be recycled for the uncertainty quantification study.

One of the most widely accepted methods to propagate uncertainty through CFD models are Polynomial Chaos methods. Carnevale et al. [1] coupled an UQ approach considering geometrical deviation in an LES simulation for internal cooling 
devices. However, their continuous Polynomial Chaos method did not perform well in the presence of discontinuities. They required very high orders for convergence, and also created oscillation in the vicinity of the discontinuity, referred to as Gibbs Phenomenon. The prevalent solution to this problem is to partition the stochastic domain into continuous sub-domains, and then separately employ continuous Polynomial Chaos to the individual sections. Such approaches are called local methods. Examples include the Multi-Element generalised Polynomial Chaos (ME-gPC) as shown in Wan and Karniadakis [2] and the Multi-Element Probabilistic Collocation method as shown in Lin and Tartakovsky [3]. Their main issue is that the position of the discontinuity has to be determined a priori. Sargsyan [4] therefore suggests the use of Bayesian inference to efficiently locate discontinuities from scarce data.

With regard to the need to automate UQ methods, Lucor [5] performed a study about adaptive methods for uncertainty quantification in transonic flows. Among them where the generalised Polynomial Chaos (gPC) method as proposed by Lucor and $\mathrm{Su}$ [6], the Padé-Legendre approximation detailed in Chantrasmi [7], Wiener-Haar Wavelets approaches by Le Mattre [8] and the Simplex Stochastic Collocation (SSC) method detailed in Witteveen [9] and Edeling [10]. Most recent works in the area move towards the strategy suggested in this work and adopt techniques from machine learning. Gorodetsky [11] created an automatic labelling procedure of different model regions using polynomial annihilation. They then trained a kernel support vector machine to recognise the discontinuously separated surfaces. While their approach proves superior to others for more than ten input parameters, it still needs a fairly high amount of model evaluations.

To reduce the number of necessary model evaluations to an absolute minimum, we therefore suggest automated approach based on the computationally least expensive method: the least squares Padé-Legendre approximation. The main novelty of our Padé algorithm is that we replace all numerical quadrature steps with equivalent least squares approximations. Our Padé-Legendre approximation can be determined using an arbitrary set of collocation points. As a global least squares method, it can fit models to discontinuous and non-linear response surfaces with a comparatively lower number of data points than other methods, see [12]. Its only disadvantage is that previous authors have encountered stability issues related to the ill-conditioning of the least squares problem from randomly chosen collocation points.

To overcome these issues, we combine the Padé approximation with a recently developed method called Optimal Quadrature Subsampling proposed by Seshadri [13]. The method is proven to significantly improve the stability of the least squares problem by using an optimal choice of points from a tensor quadrature instead of random samples. The points are chosen to ensure a good condition number of the design matrix, which often results in accurate results using a significantly lower number of model evaluations than random sampling. To further guarantee the reliability of the Padé response surface, we combine reprojection error, smoothness, and over-fitting indicators within a statistical learning approach.

To demonstrate the proposed methodology, we provide an uncertainty quantification study using our automated method for a realistic configuration of a cooling flow interacting with a shock in an HP nozzle. The channel is contoured in order to reach sonic condition in the lower region near the cooling hole. A system of shocks is generated and interacts with the cooling flow. We parametrise the geometry using a uniformly distributed diameter of the coolant channel and a similarly uniformly distributed diameter of the leading edge of the blade located in the middle of the channel. These two random parameters affect both the shock angle and local characteristic of the cooling flow. As output quantity we consider the adiabatic effectiveness $\eta$. According to Montomoli et al. [14] the typical manufacture deviations associated with the coolant and the trailing edge are respectively $10 \%$ and $20 \%$. A UQ study for geometric uncertainty for a similar arrangement of film cooling shock interaction was previously conducted by Carnevale [15] also using Padé approximations. However, the authors only used univariate approximations and could only consider the inputs separately.

The complete numerical method combining multivariate Padé Legendre expansions with Optimal Quadrature Subsampling in a statistical learning framework is described in Section 2. In section 3 we demonstrate the method using an analytical example. In section 4 we describe the set-up of the considered film cooling shock interaction CFD simulation. Finally, in section 5 we draw conclusions from the UQ study for the considered CFD test case.

\section{NUMERICAL METHOD}

In this section we detail our numerical method to obtain accurate representations of complex non-linear and discontinuous response surfaces. It comprises two innovative methods that complement each other. The first component is the multi-variate Padé approximation. As a global non-intrusive approximation method it provides a very efficient way to determine discontinuous response surfaces using a very low number of physical model evaluations. However, it has known stability issues. We therefore suggest combining it with stability regularisation method called Optimal Quadrature Subsampling. In order to do so, various adaptations are necessary. These will be described in the sections below. Finally, we use statistical learning methods to automatically ensure reliability and convergence of the combined method. To begin with, we summarise the terminology and notation necessary to determine least squares pseudospectral approximations using the matrix notation proposed by Seshadri [13]. 


\subsection{LEAST SQUARES PSEUDOSPECTRAL APPROXIMATIONS}

Let $u(\xi)$ describe the response surface of a physical model whose input uncertainty is described through the vector of random variables $\xi$. The Padé Legendre approximation conventionally relies on Legendre polynomials. We consequently restrict ourselves to random variables with uniform probability density function (PDF) $\rho(\xi)$. Other polynomial basis functions that are optimal for other PDFs are equally possible, but less well-documented in the context of Padé approximations. The function $u(\xi)$ is in $L^{2}[-1,1]$ and can be approximated through the pseudospectral approximation

$$
u(\xi) \approx \sum_{i=1}^{c(M)} \hat{p}_{i} \psi_{i}(\xi) \quad \hat{p}_{i}=\int u(\xi) \psi_{i}(\xi) \rho(\xi) d \xi
$$

The number of linear combination terms is $c(M)=\frac{(d+M) !}{d ! N !}$ for a total order polynomial expansion and $c(M)=(M+1)^{d}$ for a tensor product expansions [16]. The coefficients $\hat{p}_{i}$ can be calculated through the (possibly weighted) discrete Fourier transform

$$
\hat{p}=\Psi u \text { (unweighted) } \text { or } \hat{p}=\Psi W^{2} u \text { (weighted). }
$$

The diagonal matrix $W$ contains the weights belonging to the tensor grid collocation points $\gamma_{i}$. The matrix $\Psi$ contains the $c(M)$ orthogonal Legendre polynomials evaluated in the collocation points. Usually the points are chosen as the tensor product of univariate quadrature rules. Here, however, we aim at a more general set-up: $N$ is the number of collocation points and can be chosen arbitrarily. The matrices $\Psi$ and $W$ can be set up as

$$
\Psi=\left[\begin{array}{ccc}
\psi_{1}\left(\gamma_{1}\right) & \cdots & \psi_{c(M)}\left(\gamma_{1}\right) \\
\vdots & \ddots & \vdots \\
\psi_{1}\left(\gamma_{N}\right) & & \psi_{c(M)}\left(\gamma_{N}\right)
\end{array}\right], W=\left[\begin{array}{ccc}
\sqrt{w_{1}} & \cdots & \\
\vdots & \ddots & \vdots \\
& & \sqrt{w_{c(M)}}
\end{array}\right]
$$

The vectors $\hat{p}$ and $u$ are defined as $\hat{p}=\left[\hat{p}_{1} \cdots \hat{p}_{m}\right]$ and $u=\left[u\left(\gamma_{1}\right) \cdots u\left(\gamma_{N}\right)\right]^{T}$. Note that the polynomial coefficient vector can be truncated to $m \leq N$. In this case, fewer basis functions are fitted to the same points. Another possibility is the use of Least Angle Regression (LAR) to determine the most active set of basis functions [17]. In general, the number of model evaluation points should be equal to or larger than the number of basis functions. The authors even had some success solving underdetermined systems using least-norm solutions. This could potentially allow the use of even fewer points [18]. However, approximation errors and uniqueness need to be explored further. The multivariate polynomial basis functions $\psi_{i}$ are calculated as the product of univariate Legendre polynomials $\prod_{i=1}^{d} \psi_{I_{i, j}}\left(\xi_{i}\right) \forall j \in 1, \ldots, c(M)$. The multi-index $I_{i, j}$ is used to reference the polynomial order of the individual univariate components of the tensor product space. For example, for two random variables it can be defined as $I_{i, j}=\left[[0,0]^{T},[0,1]^{T}[1,0]^{T}[0,2]^{T}[1,1]^{T} \ldots\left[M_{1}, M_{2}\right]^{T}\right]$ for $j=0,1,2 \ldots$ $I_{i, j}$ and the corresponding number of basis functions $c(M)$ can be defined in various ways. We will use total index sets constrained by the number of subsamples used, and recommend defining $c(M)$ as the number of rows in the index matrix. For simplicity with two parameters, Chantrasmi [19] defines $c(M)=(M+1)(M+2) / 2$. By introducing the matrices

$$
A=W^{T} \Psi^{T} \quad b=W^{T} u
$$

we can solve Eq. (2) as a weighted linear least squares problem in $L^{2}$

$$
\hat{p}=\arg \min \|A p-b\|_{2}^{2} .
$$

Several authors have previously shown that computing the Fourier coefficients using least squares approximation is computationally significantly cheaper than employing quadrature techniques [20]. The drawback of this computational advantage is that some random sets of collocation points - for instance, an unlucky set of Latin-Hypercube or pseudo-random samples - can lead to a severely ill-conditioned least squares problem and consequently large approximation errors. Seshadri et al. [13] therefore proposed a new deterministic method called Optimal Quadrature Subsampling that aims at obtaining the best least squares estimate by reducing the condition number of the linear system for a given set of basis functions. 


\subsection{OPTIMAL QUADRATURE SUBSAMPLING}

Optimal Quadrature Subsampling (OQS) is a design of experiment (DoE) approach developed by Seshadri [13] that helps to find well-conditioned least squares estimates. Instead of solving the least squares problem in Eq. (5) in a set of arbitrary random points $[20,21]$ or in all available tensor grid collocation points [22], the authors suggest a sampling strategy that reduces the condition number of the design matrix $A=W^{T} \Psi^{T}$. To that end, the authors perform a low rank approximation via rank-revealing $\mathrm{QR}$ factorization of the design matrix $A$. As we assume we have $N$ physical model evaluations available, the design matrix can be approximated through

$$
A=Q_{N} R_{N} P_{N} \underbrace{+\tilde{A}_{N} P_{N}}_{\text {error }}
$$

The matrix $Q_{N}$ holds the chosen columns $N$ of $A$ and the matrix $\tilde{A}_{N}$ holds the remaining columns, which have not yet been chosen. Its Frobenius norm can be used to describe the low rank approximation error. The permutation matrix $P_{k}$ reveals which choice of collocation points will provide the best conditioned solution to the least squares approximation problem. We will denote the vector containing $P_{k}$ 's first $k$ diagonal elements as $\pi_{O Q S}$. This is the optimal subsampling index set. Subsequently, the pruned and better conditioned minimisation problem

$$
\hat{p}_{*}=\arg \min \left\|A\left(\pi_{O Q S}\right) \hat{p}-b\left(\pi_{O Q S}\right)\right\|_{2}^{2}
$$

can be solved. An elegant Matlab implementation of OQS can be found in the authors' original paper [13].

\subsection{MULTIVARIATE PADÉ APPROXIMATIONS}

A Padé approximation is a rational function of two polynomials. In a deterministic setting, it has been used as a generalisation of the Taylor series expansion to calculate an approximation of a function given its power series. In the context of Uncertainty Quantification, a Padé approximation of a function $\mathrm{u}(\xi)$ of a uniform random variable vector $\xi$ is the ratio of two Polynomial Chaos expansions $P(\xi)$ and $Q(\xi)$

$$
u(\xi) \approx \hat{M}_{\text {Pade }}(\xi)=\frac{P(\xi)}{Q(\xi)}=\frac{\sum_{j=1}^{c(M)} \hat{p}_{j} P_{j}(\xi)}{\sum_{j=1}^{c(L)} \hat{q}_{j} Q_{j}(\xi)},
$$

with numerator order $c(M)$ and denominator order $c(L)$. The coefficient vectors $\hat{p}$ and $\hat{q}$ need to be determined in order to determine a rational pseudospectral approximation of $u$. The terms $P_{j}$ and $Q_{j}$ refer to the multivariate products of the univariate Legendre series for $\xi$ ordered by the multi-index matrix $I_{i, j}$. Our multivariate Padé approximation is based on Chantrasmi [7]. It differs from Chantrasmi's in the sense that it allows the use of an arbitrary set of collocation points to combine the method with Optimal Quadrature Subsampling or an industrial database. Let $\psi_{j}\left(\xi_{1}\right)$ be a Legendre function with $M_{1}$ terms. Then, $\psi_{j}\left(\xi_{1}\right)=1, \xi_{1}, \frac{1}{2}\left(3 \xi_{1}^{2}-1\right), \frac{1}{2}\left(5 \xi^{3}-3 \xi_{1}\right)$, for $j=1,2, . ., M_{1}$. We define the discrete scalar product corresponding to $\rho(\xi)$ through numerical Gauss quadrature

$$
\langle P, Q\rangle_{M}=\sum_{i=1}^{M+1} P\left(\gamma_{i}\right) Q\left(\gamma_{i}\right) w_{i}
$$

with optimal quadrature points matrix $\Gamma$, whose rows we denote as $\gamma_{i}$ and optimal weights vector $w$. With a quadrature rule of order $M$, the integral in Eq (9) can be accurately solved for polynomials $P_{j}$, whose univariate components $\psi_{I_{i, j}}$ both have a smaller maximum order than $2 M-1$. For Legendre polynomials, the orthogonality condition of the multivariate polynomials $P_{j}$ can be obtained through the simple formula $\left\langle P_{i}, P_{j}\right\rangle=1 /((i+1 / 2)(j+1 / 2)) \delta_{i, j}$ for $i, j \in N$.

An overdetermined system of linear equations to determine $\hat{q}$ can be derived by projecting $Q u$ onto the basis functions $c(M)$ to $c(M+k)$ :

$$
\left\langle Q u, P_{j}\right\rangle_{c(M)}=\left\langle P, P_{j}\right\rangle_{c(M)}=0 \quad \forall j=c(M)+1, \ldots, c(M+k)
$$


which can be rearranged it into

$$
\left[\begin{array}{clc}
\left\langle u P_{1}, P_{c(M)+1}\right\rangle_{c(M)} & \cdots & \left\langle u P_{c(L)}, P_{c(M)+1}\right\rangle_{c(M)} \\
\vdots & \ddots & \vdots \\
\left\langle u P_{1}, P_{c(M+K)}\right\rangle_{c(M)} & \cdots & \left\langle u P_{c(L)}, P_{c(M+K)}\right\rangle_{c(M)}
\end{array}\right]\left[\begin{array}{c}
\hat{q}_{1} \\
\vdots \\
\hat{q}_{c(L)}
\end{array}\right]=0 .
$$

The index $i$ in Eq (11) runs from $c(M)$ to $c(M+k)$ because the projection error of the multi-dimensional Padé approximation onto the Legendre basis is only zero up to order $c(M)$. From $c(M)$ to $c(M+k)$ the projected error is merely minimized in the least-squares sense and the condition $c(M+k)-c(M)>c(L)$ must be uphold to ensure that $\mathrm{Eq}(11)$ is always overdetermined. The variable $k \in \mathbb{N}$ should be greater than 1 and it is beneficial for stability to keep $k \geq 2$.

As suggested by Chantrasmi [7], Eq (11) can be rewritten in an easier to implement way by introducing the three matrices

$$
\begin{aligned}
& B_{i, j}=P_{i}\left(\gamma_{i}\right) w_{j} \quad c(M)+1 \leq i \leq c(M+k), \quad 0 \leq j \leq c(M) \\
& C_{i, j}=u_{i}\left(\gamma_{i}\right) \delta_{i j} \quad 0 \leq i \leq N, \quad 0 \leq j \leq N \\
& D_{i, j}=P_{j}\left(\gamma_{i}\right) \quad 0 \leq i \leq c(M), \quad 0 \leq j \leq l
\end{aligned}
$$

The denominator design matrix $A_{q}$ is then $A_{q}=B C D$. One important detail here is that the numerical quadrature of the inner products requires the solution of $u$ in all $n$ numerical quadrature point locations. This is a temporary simplification and it will be removed in future versions of the code. However, since $Q$ is merely used to precondition the actual polynomial approximation, the caused error is not large and decreases by adding points. Based on $A_{q}$, one can find $q$ through solving the minimisation problem

$$
\min _{\|\hat{q}\|=1}\left\|A_{q} \hat{q}\right\|^{2}
$$

It can be uniquely solved using a Single Value Decomposition of $A_{q}=U S V$, where $V$ contains the eigenvalues of $A_{q}^{T} A_{q}$. The sought solution is then given by the last column of $V q=V e_{1}$, because the first eigenvalue is the smallest. The matrix $A_{q}$ is of size $(c(M+k)-c(M)) \times c(L)$. This procedure is known as generalised least-squares Padé approximation [23]. In it, however, only the denominator coefficients are found using weighted regression. The polynomial coefficients $\hat{p}$ of the numerator expansion $P(\xi)$ are calculated by employing a quadrature formula. To combine the method with Optimal Quadrature Subsampling, the coefficients need to be fitted to arbitrary points using a least squares regression. This is straightforward if the problem is regarded as a conventional non-intrusive Polynomial Chaos problem with pre-conditioner matrix $Q$ :

$$
\hat{p}=\arg \min \|\hat{p} \Psi-Q u\|_{2}^{2} .
$$

For higher generality it is useful to replace the denominator polynomial index $c(L)$ with a manual integer $L$. Using $c(L)$ can limit the model to an unnecessary high order for the denominator during the automatic fitting process. Moreover, it excludes the possibility that the best fit is continuous, which we do not want to exclude to keep the method as general as possible.

\subsection{AUTOMATIC MODEL SELECTION}

With the Least Squares Padé Approximation described above, a transonic CFD code can be evaluated in an arbitrary set of parameter collocation points. This is most useful for the industrial application of the method, where an already available database of test cases can be recycled. In addition, CFD codes do occasionally not converge in optimal quadrature locations. This issue can be easily avoided using arbitrary points. To ensure a reliable fit we use leave-one-out cross validation and solve the least squares problems using $Q R$ decompositions. When starting from a manually chosen set, we recommend adding further samples using three principles. First, the domain should be equally covered. Second, regions with high gradients are to be preferred. Third, the position of additional points should be chosen so that the condition number is reduced. If CFD convergence issues occur, it can be beneficial to interpolate to the numerically more efficient points, bearing in mind that proximity is key for accuracy. A concise summary of the most relevant lines of Matlab codes is shown below. 


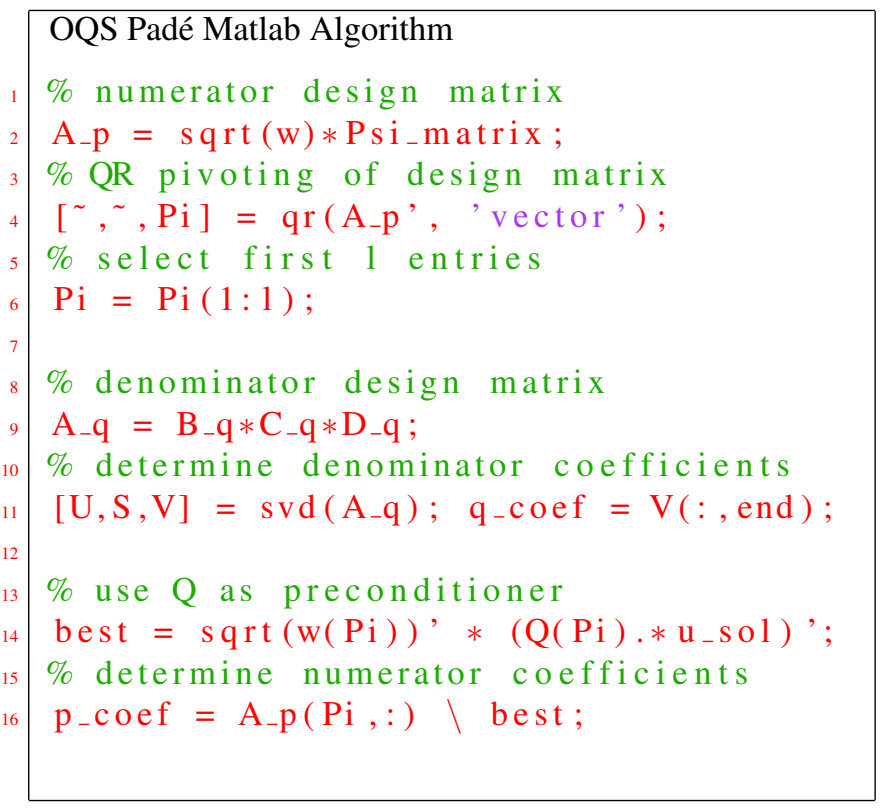

The main advantage of the least squares Padé approximation is that many numerator and denominator polynomials can be fitted to a given set of points. Therefore, criteria are needed to determine the most suitable parameters for $M$ and $L$. The goodness of fit of the surrogate model is usually evaluated using an $L_{2}$-norm error. Minimising only the reprojection error, however, can lead to the well-known problem of over-fitting. This means that the fitted model only describes the solution in the given data points, but not in between. The effect is best known for causing high amplitude oscillations between points when fitting higher order polynomials under the name of 'Runge Phenomenon'.

Over-fitting errors can be reduced by minimising the prediction error instead of the reprojection error. For a given data set, the points are divided into learning and training set, so that the model's predictive behaviour can be evaluated from training data that were not involved in the determination process. In this work, we will use Leave-one-out cross-validation. $\mathrm{K}$-fold cross validation is usually more reliable. However, since we operate with very low numbers of collocation points, we cannot afford to omit many points $[12,20]$. For the special case of linear regression, a very elegant theorem is available that allows determining the Leave-one-out error $\varepsilon_{L O O}$ in a single model fit instead of having to perform $N$ fits for $N$ points. The result can be quickly derived by extending Eq (2) using the normal equation

$$
\hat{u}=\Psi \hat{p}=\Psi\left(\Psi^{T} \Psi\right)^{-1} \Psi^{T} u=H u
$$

If the vector $h$ is defined to contain the diagonal elements of $\mathrm{H}: h=\operatorname{diag}(H)$, the Leave-one-out error can be computed as

$$
\varepsilon_{L o o}=\frac{1}{N} \sum_{i=1}^{N} \frac{u_{i}-\hat{u}_{i}}{\left(1-h_{i}\right)^{2}}
$$

To quantify unwanted discontinuous outbreaks in the response surface, Chantrasmi [19] suggested the use of the total variance as an indicator for smoothness

$$
\tau_{T V} \approx \sum_{i=1}^{N-1} \sum_{j=1}^{N-1}\left|\hat{u}_{i+1}-\hat{u}_{i}\right|
$$

Combining these indicators, we choose the optimal combination of prediction error and smoothness for a given number of points by minimising the following cost function

$$
\underset{M, L}{\arg \min }\left(\log \left(\varepsilon_{L O O}\right)+\frac{\tau_{T V}}{\max \left(\tau_{T V}\right)} \operatorname{mean}\left(\log \left(\varepsilon_{L O O}\right)\right)\right)
$$

The normalisation and multiplication by the error mean is added to ensure equal weighting of both quantities. The logarithm of the errors makes it easier for an optimisation method to identify differences. To ensure the reliability of this method in the 
presence of discontinuous, $\mathrm{Eq}(18)$ has to be complemented with an Inf and $\mathrm{NaN}$ filter. We therefore filter all values larger than 10 from the error and smoothness arrays and replace them with one to recognise them. Figure 2 shows a variety of model evaluations for different parameters of $m$ and $l$ and the final choice of the method.

\section{DEMONSTRATION USING ANALYTICAL EXAMPLE}

We demonstrate the OQS Padé Matlab Algorithm by applying it to a simple analytical example: two fairly smooth domains created by two-dimensional sinus curves divided by a sharp rising edge created by a tangens hyperbolicus function:

$$
z=\tanh \left(10 \xi_{1}\right)+0.2 \sin \left(10 \xi_{1}\right)+0.3 \xi_{2}+0.1 \sin \left(5 \xi_{2}\right)
$$

The resulting 3D surface is taken after the response surfaces obtained for the thermal efficiency in the later described film cooling shock-interaction problem. It can be seen in Figure 1 how the choice of optimal subsamples from the tensor rule allows reconstructing the previously used tanh response surface with high accuracy using only 18 collocation points. In comparison, the full tensor quadrature rule with a full 16 points misses most of the details and looks more like a linear approximation. The graph in Figure 2 shows the Pareto front for leave-one-out error and smoothness parameters. It can be
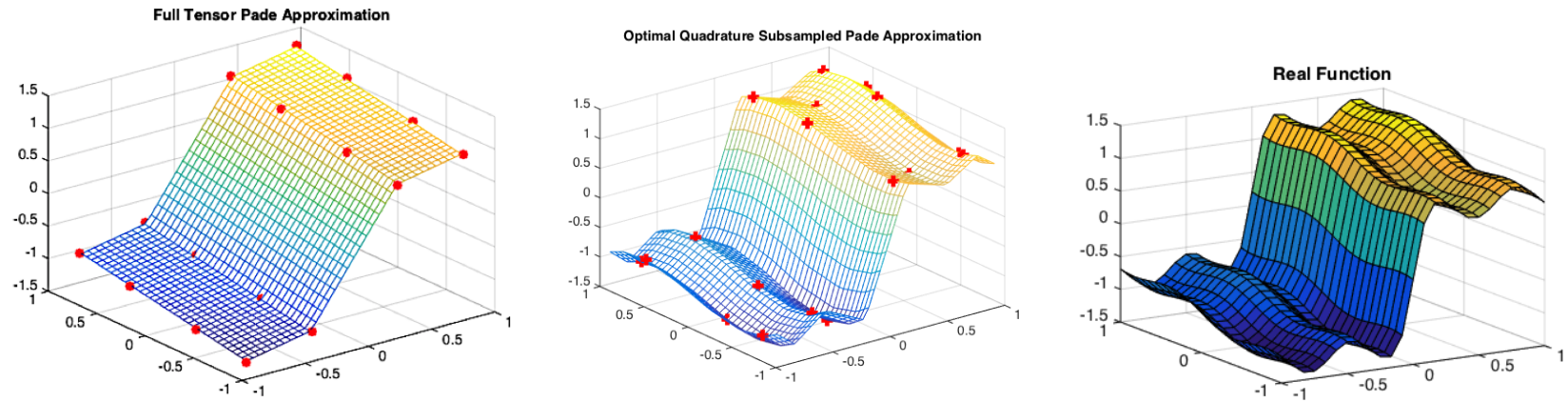

Fig. 1. Response surfaces of tangens hyperbolicus using a 16 points full tensor grid (top) and an 18 points optimally subsampled grid (bottom)

seen that the smoothness is more important for the Padé approximation than for common approximations. The error alone can be minimised even if there are singularities between the given collocation points. A reliable surface can be chosen only if the variation of the area between the points is taken into account and convergence is reached in the values. The found

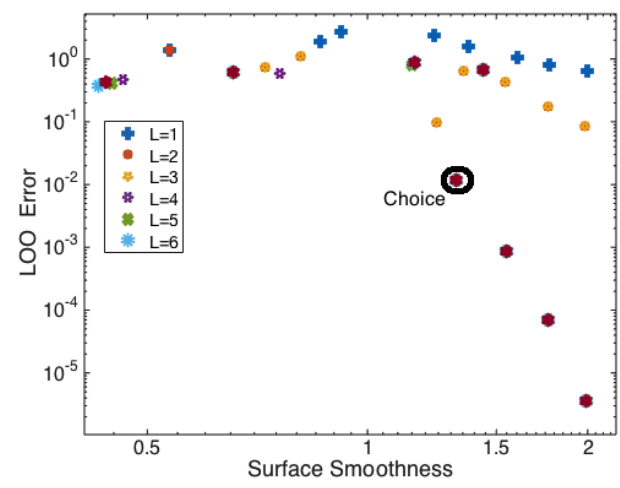

Fig. 2. Demonstration of automatic parameter selection algorithm

response surface is obtained for $[M, L, N]=[6,1,18]$. The mean and variance are estimated as $\mu=1.97 e-04$ and $\sigma=0.9476$ compared to Monte Carlo Simulation with $10^{7}$ samples of $\mu=-3.45 e-04$ and $\sigma=1.009$. The method converged because $\epsilon_{L 2}=4.43 e-15$ and $\operatorname{Err}_{L o o}=1.0778 e-59$ and the smoothness error is 1.32 . 


\section{CFD TEST CASE}

To demonstrate the above described method in a realistic framework, we apply it to series of CFD simulations previously investigated by Salvadori et al. [24]. A complete description of the experimental set-up can be found in Ochs et al. [25]. We only consider one cylindrical hole (out of five) and make the hypothesis that the flow is symmetric. The final control volume is shown in Figure $3 \mathrm{a}$ and includes the converging nozzle, the shaped plate, the cylindrical hole and the plenum. According to Garg [26] and Acharya [27], the shape of the plenum in film cooling simulations needs to be properly modelled because of its effect on the boundary layer development. Therefore, the plenum has been fully resolved and the inlet conditions for the coolant are imposed at the plenum inlet. Figure $3 \mathrm{~b}$ shows the main flow features of the present problem. The main-flow reaches sonic speed at the throat while the shape of the lower part of the contoured plate allows the flow to accelerate further to supersonic velocities generating an oblique shock wave. The shock starts at the trailing edge and impinges on the lower wall in a region a few diameters after the cooling hole exit position. The flow structures shown in Figure $3 \mathrm{~b}$ are referred to as the deterministic reference case. As previously mentioned, the test case is designed to have an injection hole positioned
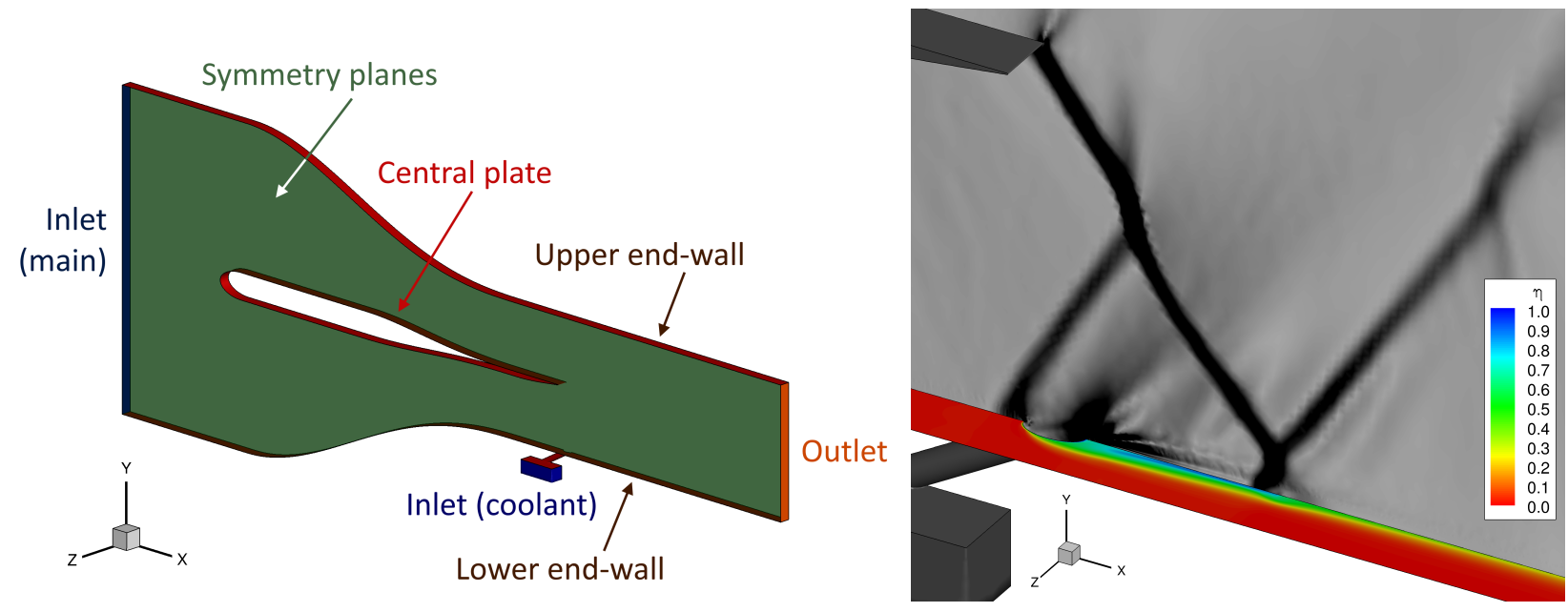

Fig. 3. (left) Control volume, (right) Flow features

upstream of the shock impingement position. The original pitch-to-diameter ratio of the five holes is 4 . The free stream Mach number at the shock location is around $M a=1.5$ (but the numerical data and the experiments suggest that it reaches higher values) and the expected blowing ratio is $M_{B}=1.0$. The non-dimensional coolant total pressure is 1.01 and nondimensional coolant total temperature is 0.557: these values are normalised with respect to the main-flow inlet total pressure and total temperature, respectively. As can be observed in Figure 3b, a weak shock is generated by the interaction between the coolant and the main-flow. This shock interacts with the oblique shock shed by the plate trailing edge that impinges on the lower wall. Furthermore, a lambda shock is generated in the impingement region. It is to be expected that the adiabatic effectiveness will be modified by the adverse pressure gradient associated with such kind of flow structure. It is usually also responsible for a local growth of the boundary layer and the insurgence of vortex structures.

The efficacy of the coolant coverage is quantified by the adiabatic effectiveness $\eta_{a w}$. It is evaluated with

$$
\eta_{a w}=\frac{T_{a w}-T_{r e c, m}}{T_{0, c}-T_{r e c, m}} .
$$

The probability distribution of $\eta_{a w}$ is the parameter considered for the UQ study described later. The adiabatic effectiveness is extracted along the centre line and compared with the experimental data for validation purposes. The distribution of the adiabatic wall temperature $T_{a w}$ is evaluated on the cooled plate by performing adiabatic calculations. The calculation of the adiabatic effectiveness requires also the evaluation of $T_{r e c, m}$ :

$$
T_{r e c, m}=T_{0, m} \frac{1+\operatorname{Pr}^{0.33 \frac{\gamma-1}{2} M a_{i s}^{2}}}{1+\frac{\gamma-1}{2} M a_{i s}^{2}} .
$$

\subsection{GRID AND TEST CASE MODELLING}

Steady Reynolds-Averaged Navier-Stokes (RANS) equations are solved with a pressure-based approach. It must be underlined that RANS methods are still widely used in industry since they are able to capture the overall phenomenology. Pressure 
and density equations are coupled and the calculation is fully second order accurate. The k- $\omega$ SST model by Menter [28] is used as turbulence closure model with compressibility effects and a low-Reynolds correction. Inlet turbulence level is set at $5 \%$ while a turbulence length scale of $1 \mathrm{~mm}$ has been considered. Viscous walls have been set as adiabatic and viscous heating has also been considered to make the heat transfer evaluation accurate. Calculation is considered to be converged when all the residuals are constantly below $10^{-4}$ and the mass-flow error is below $1 \%$. The meshes used for all the nine

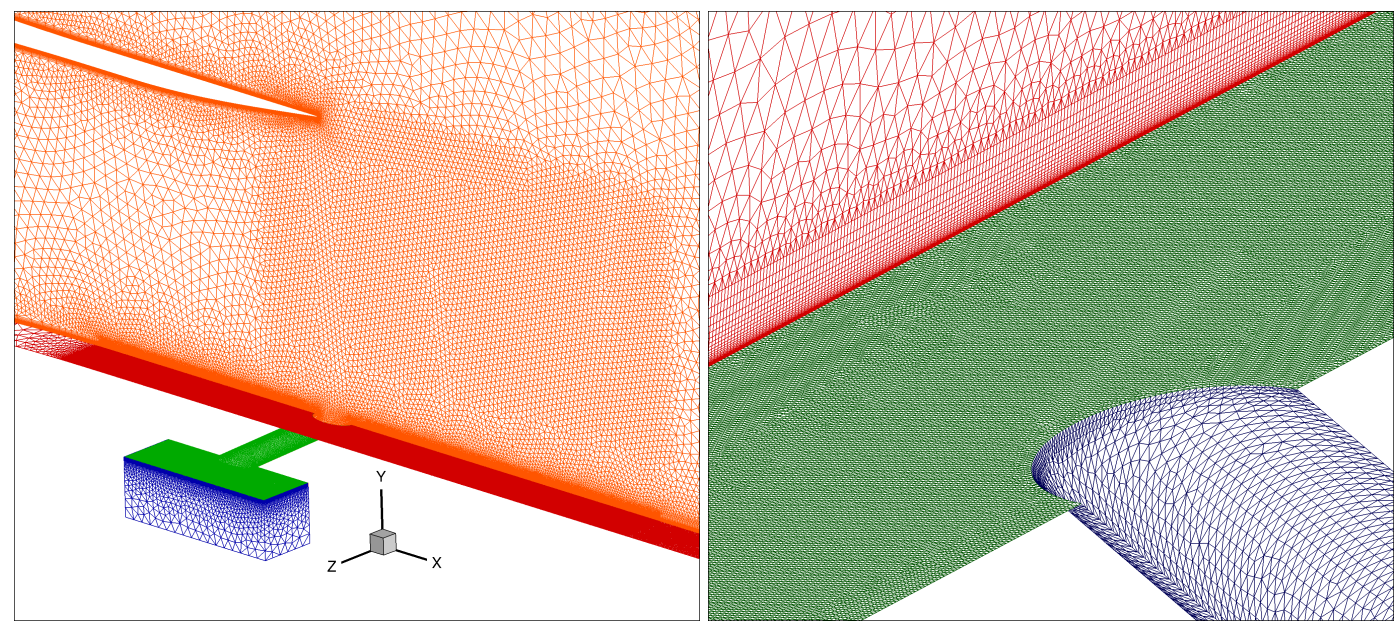

Fig. 4. Grid details in coolant/shock interaction region

calculations (necessary for the UQ analysis) are generated using the commercial hybrid grid generator Centaur $^{T M}$ by Centaursoft. The mesh is denser in the region where the shock-boundary layer interaction is expected (see Figure 4a) and the overall quality of the mesh is very high. The mean dimension of tetrahedral elements downstream of the coolant hole has been reduced to better capture the local phenomena, with a very high resolution of the lower surface (see Figure 4b). A y+ far below 1. has been obtained for the first prismatic layer and at least five prismatic elements are included in the laminar sub-layer.

\subsection{CFD RESULTS}

Figure 5 shows a comparison between the numerical and experimental maps of $\eta_{a w}$. The numerical simulation greatly underestimates the lateral spreading of the cooling flow compared to the experiment by Ochs [25].
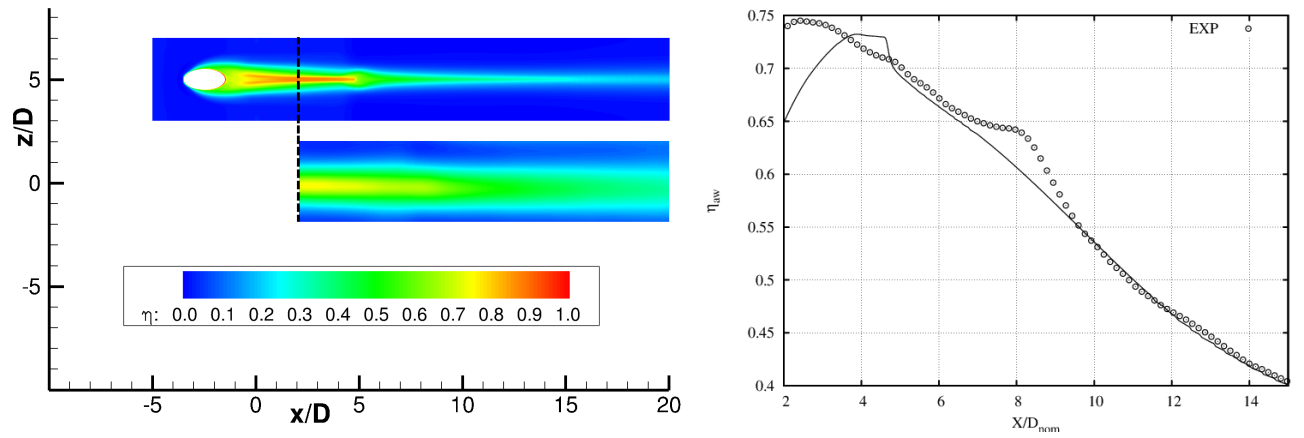

Fig. 5. (a) Comparison between CFD (upper) and experimental data (lower) for the deterministic reference configuration case. (b) $\eta_{a w}$ distribution over the centerline

The coolant flow seems to be concentrated around the centre line in the numerical simulation, whereas the experiment shows a wider redistribution of the flow coming from the hole. Figure $5 \mathrm{~b}$ compares the film cooling effectiveness distribution in stream-wise direction on the centre-line. The obtained result can be explained looking at the streamlines exiting from the coolant flow for the nominal case. In Figure 6 the isentropic Mach number distribution is shown on the coolant symmetry 
plane and lower wall and the streamlines exiting from the cooling hole. As can be observed, the cold flow is initially kept near the lower wall, coherently with the adiabatic effectiveness maps in Figure 5. A few diameter lengths later (approximately $x / D=5.0$ ), the adverse pressure gradient associated with the shock impingement generates a lift-off effect of the coolant that is visible in the smaller image in Figure 6, where the density gradient magnitude is reported. In that region the
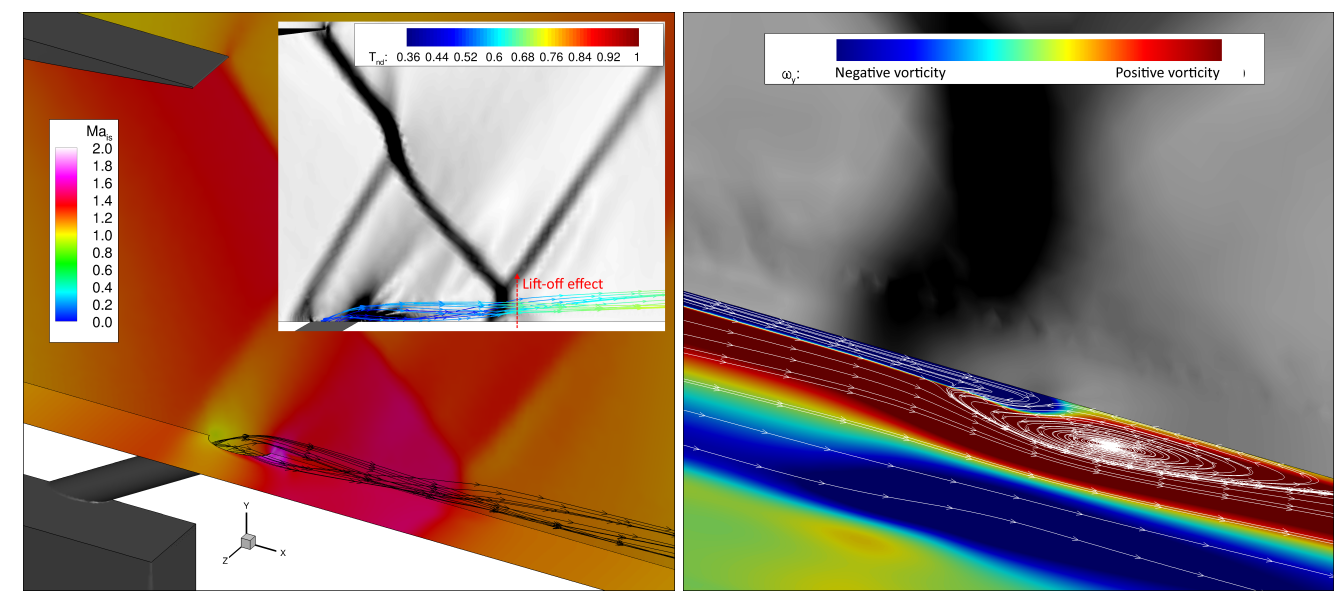

Fig. 6. (a) Flow streamlines and interaction with the shock. (b) Details of the tornado vortex after the shock

boundary layer height is increased by the shock impingement and the cooling flow is detached from the wall. After the shock interaction, an additional vortex system is generated with axis normal to the wall as show in Figure 6b. This vortex is usually named "tornado vortex". As a consequence of this vortex the coolant flow does not reattach before the end of the control volume. This explains why the cooled end-wall is so narrow in the numerically predicted adiabatic effectiveness map. A more detailed analysis of the local interaction between the main flow and the cooling flow is reported in Figure 7, where two slices perpendicular to the end-wall are reported considering a region between the hole and the shock impingement position. The streamlines are superimposed on the temperature profiles to visualize the local vortex formation and the cooling flow redistribution. At the section $x / D=-1.0$ the cooling flow is initially located in a region next to the end-wall (Figure 7a) where is affected by a strong system of recirculating vortex. This kind of vortexes are generated by the interaction of the
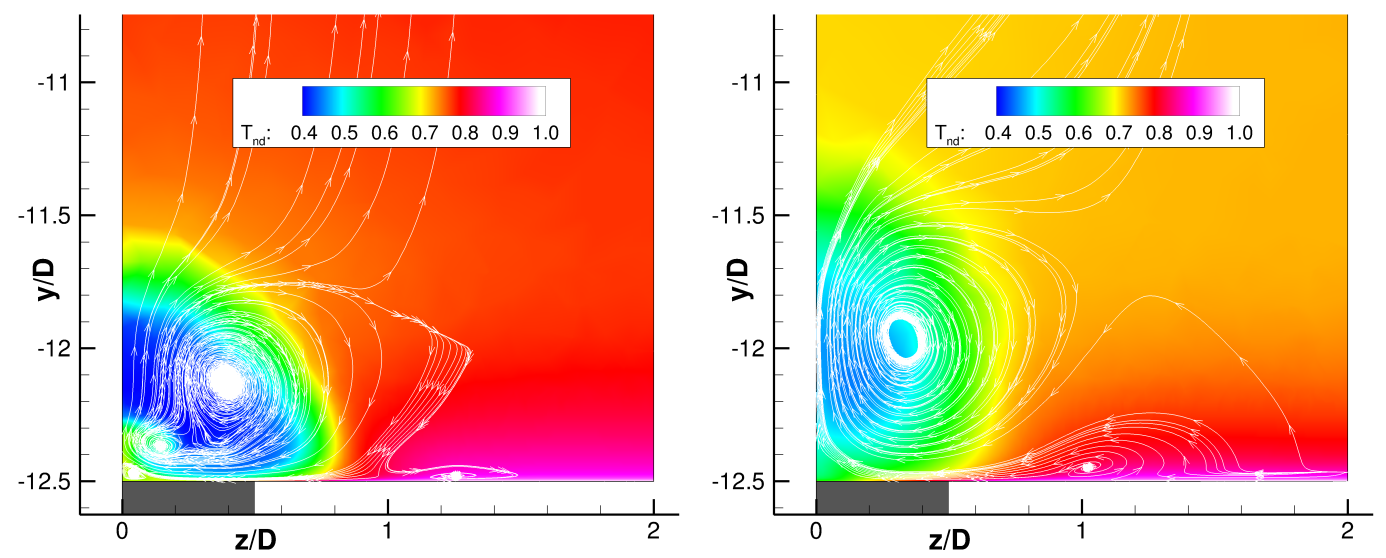

Fig. 7. Temperature maps and streamlines between hole and shock

boundary layer inside the channel flow and are usually referred to as "kidney vortex". That flow structure is responsible for the rapid movement of the coolant flow far from the wall. In a downstream location such as $x / D=2.0$, the coolant flow is completely detached from the end-wall (Figure 7b) and the hot main-flow substitutes the cold flow thus decreasing the adiabatic effectiveness value. This mechanism is strongly dependent by geometrical parameters such as the coolant diameter. The dimension of the kidney vortex is affected by shape and the dimension of the coolant hole. The interactions with the 
shocks and their angle are affected by the trailing edge of the next blade. All described flow features are coherent with the previously observed tornado vortex phenomenon, which according to Hagen and Kurosaka [29], Carnevale et al. [15] and recently Zhou et al. [30], is responsible for the local reduction of the cooling effectiveness.

\section{UQ RESULTS}
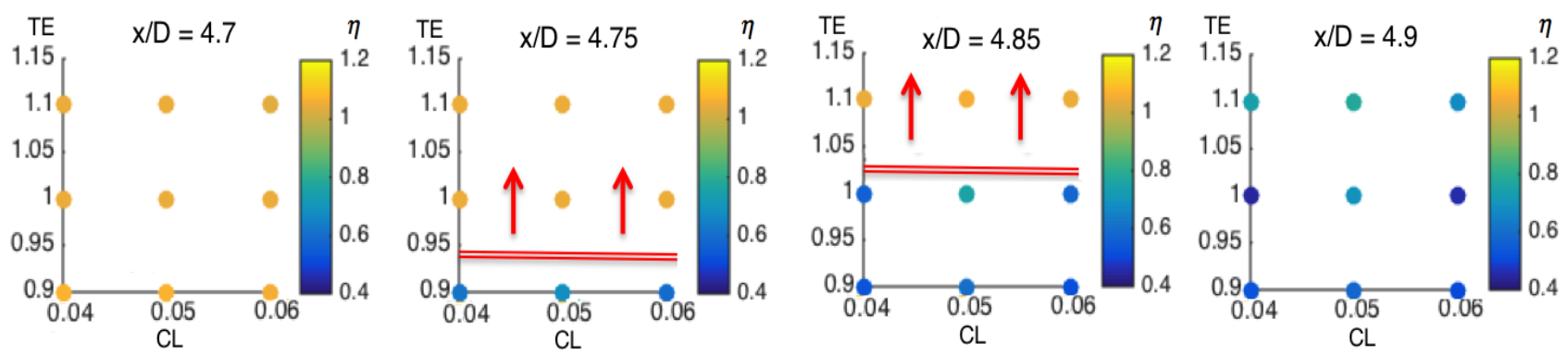

Fig. 8. The graphs illustrates how the shock moves through the stochastic response surface of the thermal efficiency.

In this UQ study, we consider the above described CFD case dependent on two random variables: a uniformly distributed diameter of the coolant channel $\xi_{1} \sim U[0.9,0.1]$ and a similarly uniformly distributed diameter of the leading edge of the blade located in the middle of the channel $\xi_{2} \sim U[0.04,0.06]$. Using the adiabatic effectiveness $\eta$ as output quantity, we try to reconstruct three dimensional discontinuous response surfaces resulting from this two parameter uncertainty using the newly proposed methodology.

A test matrix of 9 deterministically run CFD cases was already available. The top four graphs in Figure 8 show the test matrix at four different $x$-locations, which are the nine different combinations of the three values of trailing edge diameters $[0.9,1.0,1.1]$ and cooling hole diameters $[0.04,0.05,0.06]$. Naturally, an execution of the model in the optimal sub-sample quadrature point locations would have been much preferable. However, with the idea in mind to recycle already available CFD simulation data, we took the number of these 9 deterministic simulation as a constraint.

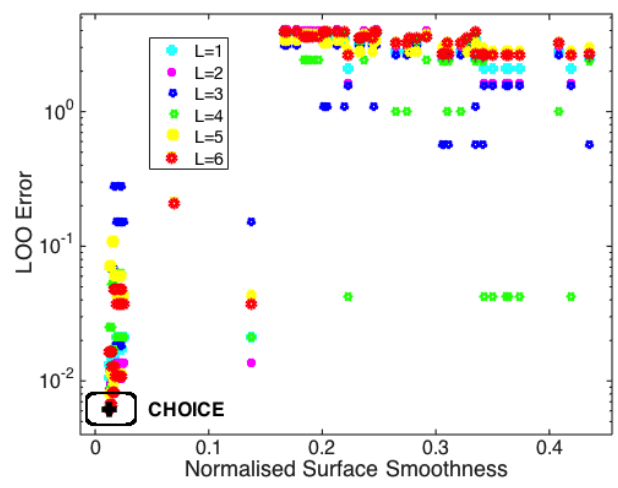

Fig. 9. Example of found Pareto front and choice of the automatic response surface error and smoothness control

The four graphs in Figure 8 show how the discontinuity moves from the bottom to the top of the stochastic domain between $x / D=4.7$ and $x / d=4.9$. At $x / D=4.7$ all values of the response function have nearly the same level. In the graph right next to it, however, at $x / D=4.75$ a clear drop can be seen between two domains: one levelled domain at the top and another levelled domain at the bottom at a trailing edge diameter of 0.9. The same drop in the stochastic domain can be observed for trailing edge diameter values of $0.9-1$ at $x / D=4.85$. The location $x / D=4.9$ is located after the shock so that the domain is at nearly at one level again.

Because the CFD mesh in the shock region is extra fine, hundreds of such discontinuous shock responses have to be fitted automatically and reliably. To that end, the automated multivariate Padé approximation described above is used. Various orders of $M$ and $L$ are automatically tested and fitted to the available CFD points. All parameter possibilities for one response 
surface are displayed in Figure 9. In the considered example a clear minimum is found by the method. In other cases a Pareto front is found and an equally weighted trade-off between the two objectives is made. The graphs in Figure 11 show three examples of surfaces that where automatically fitted by the approach.
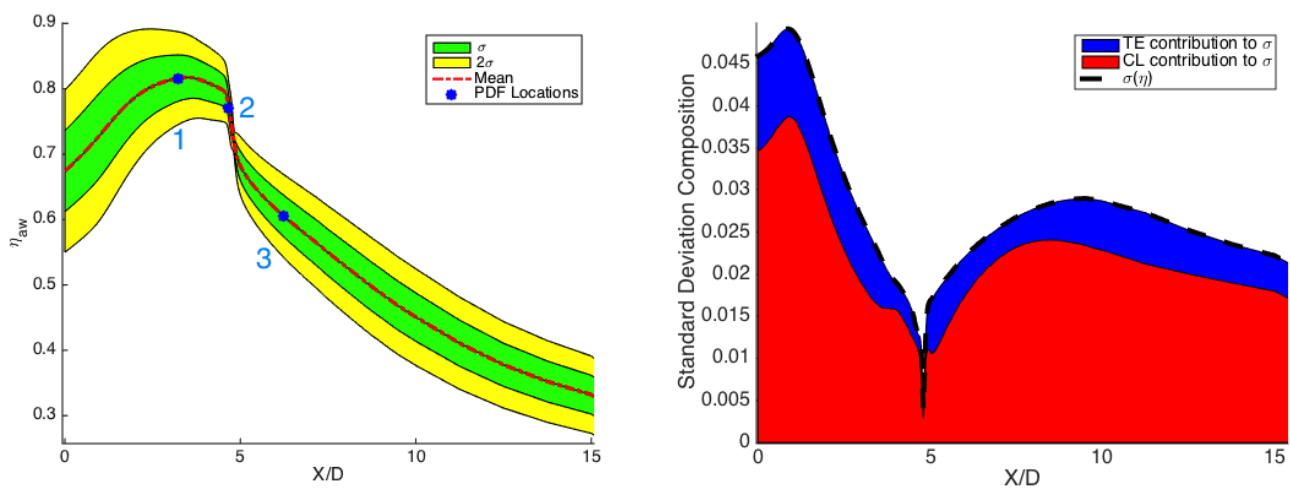

Fig. 10. Mean and standard deviation of the film cooling effectiveness (top) and sensitivity analysis of input parameters using Sobol Indices (bottom)
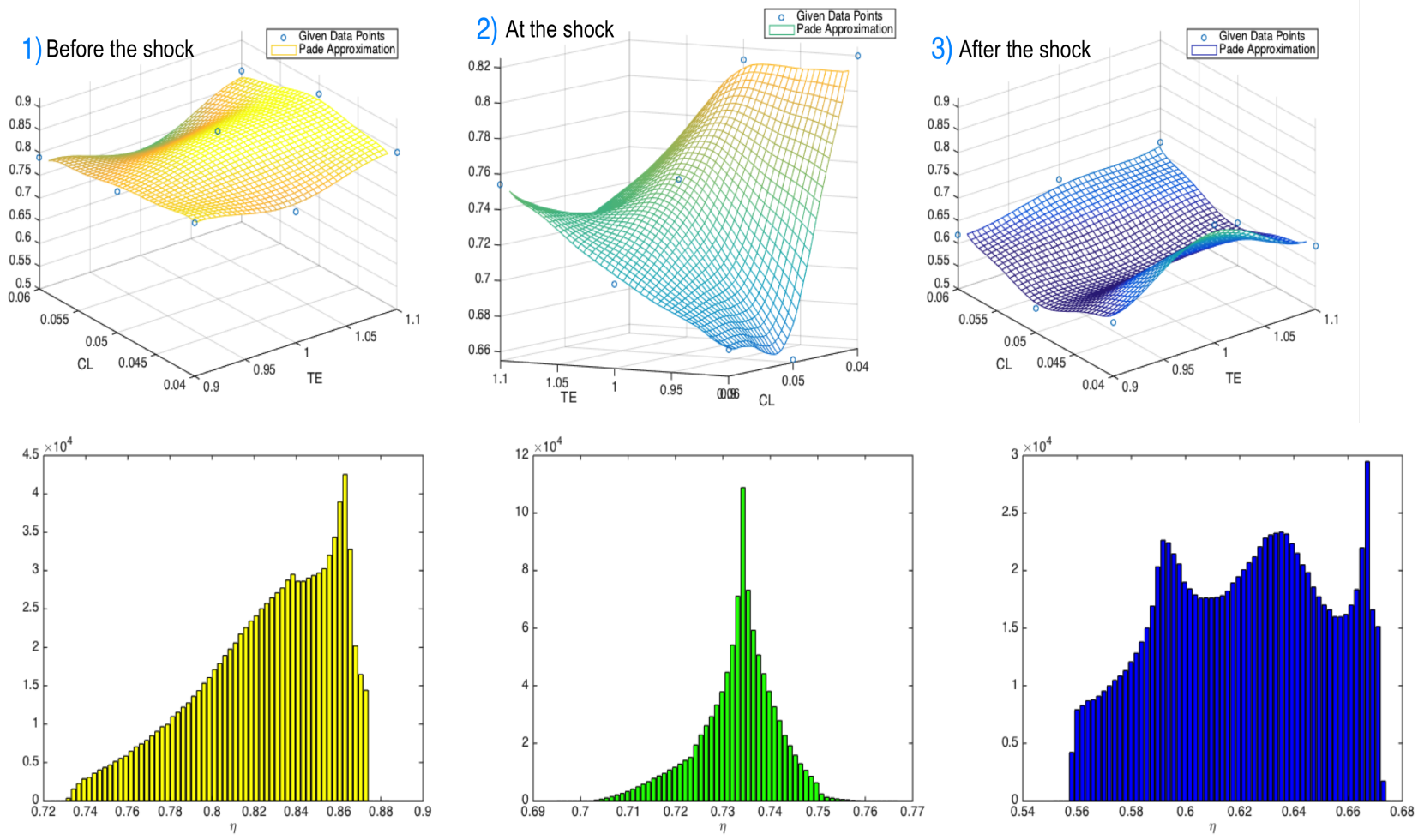

Fig. 11. Three examples of automatically fitted Padé response surfaces before (1), at (2) and after (3) the shock location. The bottom row shows three PDFs to the $\mathrm{x}$-locations before, at and after the shock.

Throughout the CFD domain, not only discontinuous but in absence of shocks also smooth response surfaces occur. The smooth response surfaces before (1) and after (3) the shock in Figure 11 were fitted by the method with a denominator order of $L=1$. This means that no discontinuity was found, which of course agrees with the physical data. It shows that the Padé approximation is also quite suitable for lower order approximations of continuous response surfaces. At the shock locations the value was mostly $L=2$. Thus, only a single discontinuity was found by the method for certain values of the trailing 
edge diameter. The probability distributions found from these model evaluations are shown in the bottom row of Figure 11. They show the following: before the shock, uncertainty in the diameters is twice as likely to cause a higher thermal efficiency than a lower one. The shock location itself is only weakly influenced by the uncertainty. It remains stationary with a high probability. However, the resulting distribution is clearly fat-tailed. After the shock, the distribution seems to be in three states with peaks at $0.59,0.63$ and 0.67 .

After fitting response surfaces to all $x$ locations the Polynomial Chaos coefficients of the expansion are used to a) calculate mean and standard deviation as shown in the top graph of Figure 10 and b) the Sobol indices to understand to what proportions the diameter of the trailing edge and cooling holes contribute to the experienced uncertainty. The results, shown in the bottom graph of Figure 10, are quite coherent with the authors' expectation that it should be the cooling whole diameter which is more significant. An interesting observation made from this sensitivity analysis is that the uncertainty in the output is significantly reduced at the shock location. The bottom graph of Figure 10 shows that at $x / D=4.8$ the standard deviation goes almost down to zero. Another interesting observation is that the cooling effect is strongly influenced by the shock. It sharply decreases before the occurrence of the shock and increases afterwards. The trailing edge diameter on the other hand, seems to have more of a constant effect on the standard deviation.

\section{CONCLUSION}

Although Padé approximations are known for their instability, contributions made in this work have shown that least squares multivariate Padé approximations can be an efficient and reliable automatic tool for uncertainty quantification in CFD. We have improved the accuracy and reliability of Padé approximations by combining them with Optimal Quadrature Subsampling to reduce least squares estimation errors and statistical learning techniques to indicate error, smoothness, and over-fitting. Using a cost function approach, an algorithm was developed to choose the most suitable response surface automatically. The combined methodology can accurately describe stochastic responses of transonic flow simulations, even if they are highly non-linear and contain discontinuities with a very low number of necessary CFD model evaluations. Moreover, the new combination of optimal subsampling and multivariate Padé approximation allows the reduction of the number of necessary flow simulations for discontinuous uncertainty quantification problems to a new minimum. To demonstrate the developed method in a real-world engineering setting, it has been applied to a 3D film cooling shock interaction problem. The challenge of this problem is that, due to the fine grid in the vicinity of the shocks, thousands of discontinuous stochastic response surfaces have to be fitted automatically to perform accurate uncertainty quantification. It was shown that the method achieved this smoothly and with a low error. Moreover, it could be established that the cooling hole diameter is significantly

more important than the trailing edge diameter. The general approach can be easily applied to a wide range of other case studies representative of real engine effects.

\section{ACKNOWLEDGEMENTS}

The authors would like to acknowledge the European Commission and the TATEF2 project consortium for the research. The authors would also like to thank the University of Karlsruhe for sharing their data and Prof Martelli of the University of Florence for his contribution.

\section{References}

[1] Carnevale, M., Montomoli, F., D’Ammaro, A., Salvadori, S., and Martelli, F., 2013. “Uncertainty Quantification: A Stochastic Method for Heat Transfer Prediction Using LES”. Journal of Turbomachinery, 135(5), jun, p. 051021.

[2] Wan, X., and Karniadakis, G. E., 2005. "An adaptive multi-element generalized polynomial chaos method for stochastic differential equations". Journal of Computational Physics, 209(2), pp. 617-642.

[3] Lin, G., and Tartakovsky, A. M., 2007. "An efficient, high-order multi-element probabilistic collocation method on sparse grids for three-dimensional flow in random porous media". In AGU Fall Meeting Abstracts, Vol. 1, p. 1318.

[4] Sargsyan, K., Safta, C., Debusschere, B., and Najm, H., 2012. "Uncertainty Quantification given discontinuous model response and a limited number of model response evaluations". SIAM Journal on Scientific Computing, 34(1), pp. 4464.

[5] Lucor, D., a.S. Witteveen, J., Constantine, P., Schiavazzi, D., and Iaccarino, G., 2012. "Comparison of adaptive uncertainty quantification approaches for shock wave-dominated flows". Center For Turbulence Research, Prooceedings of the Summer Program 2012, pp. 219-228.

[6] Lucor, D., Su, C.-H., and Karniadakis, G. E., 2004. "Generalized polynomial chaos and random oscillators". International Journal for Numerical Methods in Engineering, 60(3), pp. 571-596.

[7] Chantrasmi, T., Doostan, A., and Iaccarino, G., 2009. "Padé-Legendre approximants for uncertainty analysis with discontinuous response surfaces". Journal of Computational Physics, 228(19), pp. 7159-7180. 
[8] Le Maitre, O. P., Knio, O. M., Najm, H. N., and Ghanem, R. G., 2004. "Uncertainty propagation using Wiener-Haar expansions". Journal of computational Physics, 197(1), pp. 28-57.

[9] Witteveen, J. A. S., and Iaccarino, G., 2012. "Simplex stochastic collocation with random sampling and extrapolation for nonhypercube probability spaces". SIAM Journal on Scientific Computing, 34(2), pp. A814-A838.

[10] Edeling, W. N., Dwight, R. P., and Cinnella, P., 2016. "Simplex-stochastic collocation method with improved scalability". Journal of Computational Physics, 310, pp. 301-328.

[11] Gorodetsky, A., and Marzouk, Y., 2014. "Efficient Localization of Discontinuities in Complex Computational Simulations". SIAM Journal on Scientific Computing, 36(6), pp. A2584-A2610.

[12] Sudret, B., Marelli, S., and Lataniotis, C., 2015. "Sparse Polynomial Chaos Expansions as a Machine Learning Regression Technique". In International Symposium on Big Data and Predictive Computational Modeling.

[13] Seshadri, P., Narayan, A., and Mahadevan, S., 2016. "Optimal Quadrature Subsampling for Least Squares Polynomial Approximations". arXiv preprint arXiv:1601.05470.

[14] Montomoli, F., Carnevale, M., D’Ammaro, A., Massini, M., and Salvadori, S., 2015. Uncertainty Quantification in Computational Fluid Dynamics and Aircraft Engines. Springer, Berlin.

[15] Carnevale, M., D’Ammaro, A., Montomoli, F., and Salvadori, S., 2014. "Film Cooling and Shock Interaction: An Uncertainty Quantification Analysis With Transonic Flows". In ASME Turbo Expo 2014: Turbine Technical Conference and Exposition, American Society of Mechanical Engineers, pp. V05BT13A001-V05BT13A001.

[16] Bungartz, H.-J., and Griebel, M., 2004. "Sparse grids". Acta numerica, 13, pp. 147-269.

[17] Blatman, G., and Sudret, B., 2011. "Adaptive sparse polynomial chaos expansion based on least angle regression". Journal of Computational Physics, 230(6), pp. 2345-2367.

[18] Donoho, D. L., 2006. "For most large underdetermined systems of linear equations the minimal 11-norm solution is also the sparsest solution". Communications on pure and applied mathematics, 59(6), pp. 797-829.

[19] Chantrasmi, T., Iaccarino, G., Najm, H. N., and Papanicolaou, G., 2011. Padé-Legendre method for uncertainty quantification with fluid dynamics applications. Stanford University.

[20] Palar, P. S., Tsuchiya, T., and Parks, G. T., 2016. "Multi-fidelity non-intrusive polynomial chaos based on regression". Computer Methods in Applied Mechanics and Engineering, 305, pp. 579-606.

[21] Hosder, S., Walters, R. W., and Balch, M., 2007. "Efficient sampling for non-intrusive polynomial chaos applications with multiple uncertain input variables". Collection of Technical Papers - AIAA/ASME/ASCE/AHS/ASC Structures, Structural Dynamics and Materials Conference, 3(April), pp. 2946-2961.

[22] Loeven, A., and Bijl, H., 2009. "An Efficient Framework for Uncertainty Quantification in CFD Using Probabilistic Collocation”. 50th AIAA/ASME/ASCE/AHS/ASC Structures, Structural Dynamics, and Materials Conference(May).

[23] Guillaume, P., Huard, A., and Robin, V., 1998. "Generalized multivariate Padé approximants". Journal of Approximation Theory, 95(2), pp. 203-214.

[24] Salvadori, S., Montomoli, F., and Martelli, F., 2013. "Film-cooling performance in supersonic flows: effect of shock impingement". Proceedings of the Institution of Mechanical Engineers, Part A: Journal of Power and Energy, pp. 295305.

[25] Ochs, M., Schulz, A., and Bauer, H.-J., 2007. "Investigation of the influence of trailing edge shock waves on film cooling performance of gas turbine airfoils". In ASME Turbo Expo 2007: Power for Land, Sea, and Air, American Society of Mechanical Engineers, pp. 465-474.

[26] Garg, V. K., and Rigby, D. L., 1999. "Heat transfer on a film-cooled blade-effect of hole physics". International Journal of Heat and Fluid Flow, 20(1), pp. 10-25.

[27] Acharya, S., and Leedom, D. H., 2013. "Large eddy simulations of discrete hole film cooling with plenum inflow orientation effects". Journal of Heat Transfer, 135(1), p. 11010.

[28] Menter, F. R., Langtry, R., and Völker, S., 2006. "Transition modelling for general purpose CFD codes". Flow, turbulence and combustion, 77(1-4), pp. 277-303.

[29] Hagen, J. P., and Kurosaka, M., 1993. "Corewise cross-flow transport in hairpin vortices - The tornado effect'". Physics of Fluids A: Fluid Dynamics (1989-1993), 5(12), pp. 3167-3174.

[30] Zhou, C., 2015. "Thermal Performance of Transonic Cooled Tips in a Turbine Cascade". Journal of Propulsion and Power, 31(5), pp. 1268-1280. 